\title{
Membrane Dynamics Induced by a Phosphatidylinositol 3,4,5-Trisphosphate Optogenetic Tool
}

\author{
Yoshibumi Ueda, ${ }^{* 1, * 2 \dagger}$ Tatsuhito II, ${ }^{* 3}$ Yuki Aono, ${ }^{* 1}$ Naotoshi Sugimoto, ${ }^{* 4}$ Seiichi ShInJI, ${ }^{* 5}$ \\ Hiroshi YoshIDA, ${ }^{* 5}$ and Moritoshi SATO*1 \\ *1 Graduate School of Arts and Sciences, The University of Tokyo, 3-8-1 Komaba, Meguro, Tokyo 153-8902, Japan \\ *2 AMED-PRIME, Japan Agency for Medical Research and Development, Tokyo, Japan \\ *3 Department of Veterinary Pathology, School of Veterinary Medicine, Nippon Veterinary and Life Science \\ University, 1-7-1 Kyonan-cho, Musashino, Tokyo 180-8602, Japan \\ *4 Department of Physiology, Graduate School of Medical Science, Kanazawa University, Kanazawa, Ishikawa, \\ Japan \\ *5 Department of Gastrointestinal and Hepato-Biliary-Pancreatic Surgery, Nippon Medical School, Bunkyo, \\ Tokyo 113-8603, Japan
}

\begin{abstract}
Membrane dynamic structures such as filopodia, lamellipodia, and ruffles have important cellular functions in phagocytosis and cell motility, and in pathological states, such as cancer metastasis. Phosphatidylinositol 3,4,5-trisphosphate (PIP $\left.\mathrm{P}_{3}\right)$ is a crucial lipid that regulates $\mathrm{PIP}_{3}$ dynamics. Investigations of how $\mathrm{PIP}_{3}$ is involved in these functions have mainly relied on pharmacological interventions, and therefore have not generated detailed spatiotemporal information concerning membrane dynamics upon $\mathrm{PIP}_{3}$ production. In the present study, we applied an optogenetic approach using the CRY2CIBN system. Using this system, we revealed that local $\mathrm{PIP}_{3}$ generation induced directional cell motility and membrane ruffles in COS7 cells. Furthermore, combined with structured illumination microscopy (SIM), membrane dynamics were investigated with high spatial resolution. We observed PIP $_{3}$-induced apical ruffles and unique actin fiber behavior in that a single actin fiber protruded from the plasma membrane was taken up into the plasma membrane without depolymerization. This system has the potential to investigate other high-level cell motility and dynamic behaviors, such as cancer cell invasion and wound healing with high spatiotemporal resolution, and could provide new insights of biological sciences for membrane dynamics.
\end{abstract}

Keywords Lipid, phosphatidylinositol 3,4,5-trisphosphate, $\mathrm{PIP}_{3}$, optogenetics, cryptochrome 2, lamellipodia, structured illumination microscopy

(Received September 20, 2018; Accepted October 23, 2018; Advance Publication Released Online by J-STAGE November 2, 2018)

\section{Introduction}

Membrane dynamics plays important roles in various cellular functions. Filopodia and lamellipodia are essential for cell motility, such as in wound recovery, axon guidance in neurons, and cancer progression. Ruffles are crucial for receptor internalization and phagocytosis. ${ }^{1}$ These membrane dynamic structures are controlled by actin polymerization/ depolymerization. Currently, a wide spectrum of signaling molecules that regulate actin dynamics have been identified, such as small $\mathrm{G}$ proteins ${ }^{2}$ and lipids. ${ }^{3}$

Phosphatidylinositol 3,4,5-trisphoshpate $\left(\mathrm{PIP}_{3}\right)$ is a functional lipid, which is among the most identified molecules that regulate a wide spectrum of cellular functions, such as lamellipodia ${ }^{4}$ and ruffles $^{5}$ in adherent cells, and the function and structure of synapses in neuronal cells. ${ }^{6-8}$ The $\mathrm{PIP}_{3}$ level is extremely low in a single cell, which is estimated to be 10000 in the static state. ${ }^{6,9}$

† To whom correspondence should be addressed.

E-mail: yoshibumiueda@gmail.com
In response to hormones and neurotransmitters, $\mathrm{PIP}_{3}$ is produced from phosphatidylinositol 4,5-bisphosphate $\left(\mathrm{PIP}_{2}\right)$ by phosphatidylinositol 3-kinase (PI3K). The extent of $\mathrm{PIP}_{3}$ production varies among cell types and hormones applied. For instance, $\mathrm{PIP}_{3}$ increases 14-fold more than that with the serumstarved condition in response to platelet-derived growth factor (PDGF) in U87MG cells. ${ }^{10}$ Epidermal growth factor (EGF) stimulation resulted in a 2 -fold $\mathrm{PIP}_{3}$ increase (about 0.3 pmol increase per $6 \times 10^{6}$ cells) in A431 cells under a serum-free condition. ${ }^{9}$ These change in the $\mathrm{PIP}_{3}$ level recruits effector proteins including Akt, ${ }^{11}$ WASP family Verprolin-homologous protein (WAVE) ${ }^{4}$ and GEF of small G proteins ${ }^{3,5,12,13}$ to local sites on the plasma membrane, resulting in the induction of membrane dynamics.

Previous studies investigating how $\mathrm{PIP}_{3}$ is required for membrane dynamics have relied on indirect approaches using inhibitors ${ }^{7,14,15}$ and RNA interference (RNAi) for $\mathrm{PI}^{2} \mathrm{~K}^{16}$ to decrease endogenous $\mathrm{PIP}_{3}$ or stimulate its production. However, these methods often underestimate the contribution of $\mathrm{PIP}_{3}$ because of the confounding activities of other signaling molecules. Additionally, long-term perturbations by knockdown 
using RNAi or overexpression of constitutively active PI3K have chronic effects that can cause secondary phenomena or cell compensation, leading to unexpected cellular changes. Therefore, the details of membrane dynamics have not been fully elucidated.

Recently, optogenetic approaches using photoreceptors derived from plants, fungi, and bacteria have emerged. ${ }^{17}$ Several photoreceptors, including phototropin1, cryptochrome 2 (CRY2), and phytochrome B (PhyB), have been harnessed as photoswitches, and they have also been intensively engineered. We have developed several optogenetic tools for apoptosis based on Vivid, a fungal blue-light photoreceptor, ${ }^{18}$ PhyB-PIF6-based $\mathrm{G}$ protein-coupled receptors, ${ }^{19}$ a transcription system based on the CRY2-CIB1 system, ${ }^{20}$ and the Magnet system ${ }^{21}$ that we developed from Vivid. These photoswitches enable us to regulate the activity of signaling molecules of interest with high spatial and temporal resolutions, and therefore overcome the limitations of previous methods. In the present study, we constructed an optogenetic tool to increase $\mathrm{PIP}_{3}$ based on the CRY2-CIB1 system, termed as PPAP ( $\mathrm{PIP}_{3}$ production by photo-activated PI3K). Using this photoswitch, we found that $\overline{\mathrm{PIP}}_{3}$ controls ruffle formation and directional cell motility. Combined with structured illumination microscopy (SIM), we were able to reveal $\mathrm{PIP}_{3}$-induced actin and membrane dynamics with a high spatiotemporal resolution in living cells. Thus, we demonstrated the usefulness of PPAP for inducing membrane dynamics. PPAP combined with SIM imaging is also applicable to revealing detailed membrane dynamics by a $\mathrm{PIP}_{3}$ increase during cancer cell invasion and in dendritic spines of neurons.

\section{Experimental}

\section{Constructs}

cDNAs encoding CRY2PHR, CIBN, P2A/T2A, tandem PH domains derived from Akt, and iRFP with codons optimized for mammalian expression were synthesized by GenScript (Piscataway, NJ). Constructed PPAP1.0 was subcloned into a pCAG vector that contains the cytomegalovirus early enhancer element fused to the chicken $\beta$-actin promoter, and the splice acceptor of the rabbit $\beta$-globin gene. ${ }^{22}$

\section{Cell culture and transfection}

HEK293, NIH3T3, and COS7 cells were cultured in Dulbecco's Modified Eagle Medium (DMEM; Nacalai) supplemented with $10 \%(\mathrm{v} / \mathrm{v})$ fetal bovine serum, penicillin $(50 \mathrm{U} / \mathrm{mL})$, and streptomycin $(50 \mathrm{mg} / \mathrm{mL})$ at $37^{\circ} \mathrm{C}$ in $5 \% \mathrm{CO}_{2}$. Cells were plated onto glass bottom dishes, transiently transfected with Lipofectamine 3000 reagent (Thermo Fisher Scientific, US) according to the manufacturer's instructions, and incubated for $24 \mathrm{~h}$ at $37^{\circ} \mathrm{C}$ in $5 \% \mathrm{CO}_{2}$.

\section{Reagents}

LY294002 was from Cayman Chemical Company (Ann Arbor, MI). cOmplete ${ }^{\mathrm{TM}}$ Protease Inhibitor Cocktail was purchased from Roche (Germany). Phospho-Akt1 antibody (Ser 473) (sc293125) and Akt1 antibody (sc-5298) were from Santa Cruz Biotechnology, CA. Triton X-100 was purchased from Kanto Chemical Co., Inc (Tokyo, Japan).

\section{Western blotting}

COS7 cells were plated onto 6-well plates. PAPP1.0 was transiently transfected using Lipofectamine 3000 according to the manufacturer's instructions, and incubated for $24 \mathrm{~h}$ at $37^{\circ} \mathrm{C}$ in $5 \% \mathrm{CO}_{2}$. One day after transfection, cells were continuously irradiated for $10 \mathrm{~min}$ with $1.0 \mathrm{~W} / \mathrm{m}^{2}$ blue light by using a LED light source (470 $\pm 20 \mathrm{~nm}$; CCS, Kyoto, Japan). LY294002 was pre-incubated $10 \mathrm{~min}$ before blue light irradiation. Then, $50 \mathrm{ng} / \mathrm{mL}$ PDGF was added for $10 \mathrm{~min}$. Cells were scraped with $750 \mu \mathrm{L}$ PBS(-) including $0.1 \%$ triton $\mathrm{X}-100$ and protease inhibitor cocktail $(\times 100)$ (Roche) at $4^{\circ} \mathrm{C}$. Then, $250 \mu \mathrm{L}$ of an SDS sample buffer was added to these samples. After being incubated with $95^{\circ} \mathrm{C}$ for $5 \mathrm{~min}$, a $10-\mu \mathrm{g}$ amount of the total protein in each well of SDS-PAGE gel was loaded. The samples were run with a current clump for around $30 \mathrm{~mA} 1 \mathrm{~h}$. The gels were transferred to a membrane with $20 \mathrm{~V}$ for $1 \mathrm{~h}$. After incubation with Blocking one (Nacalai Tesque, Kyoto, Japan) for $1 \mathrm{~h}$ at room temperature in order to block non-specific immunological reactions, first antibody for anti phospho-Akt1 antibody (Ser 473) (sc-293125: Santa Cruz Biotechnology, CA) and Akt1 antibody (sc-5298: Santa Cruz Biotechnology, CA) were added (1:1000), and incubated overnight at $4{ }^{\circ} \mathrm{C}$. Next, HRP-conjugated goat anti-mouse IgG 2nd antibody was applied (1:2000). Chemiluminescence images were taken by LAS-3000 mini (Fujifilm Corporation, Tokyo, Japan). Quantification of the signals was performed by using ImageJ software (NIH).

\section{Confocal laser scanning microscopy}

The COS7 cells were plated on glass-based dishes. One day after culturing, PPAP1.0 and $\mathrm{PH}(2 \times)$-iRFP were transiently transfected with Lipofectamine 3000 (Invitrogen). On the next day, the fluorescence of each protein was observed with a PlanApochromat $63 \times / 1.40$ Oil DIC M27 objective lens equipped to laser scanning confocal microscopy (LSM710, Carl Zeiss, Germany). To activate PPAP1.0, the region of interest in a blue square was irradiated for $126 \mu \mathrm{s} /$ pixel with a Multi-line Argon laser $(458 \mathrm{~nm})$ at $0.5 \mathrm{~W} / \mathrm{m}^{2}$, and then an image was continuously taken. The $0.5 \mathrm{~W} / \mathrm{m}^{2}$ blue light power is roughly estimated to be 1.5-times stronger than blue light $(<505 \mathrm{~nm})$ in room light. This power is much enough to induce membrane dynamics by PPAP within $5 \mathrm{~min}$, but was not toxic for cells in the present study.

\section{Structured illumination microscopy}

PPAP1.1 and lifeact-mCherry were transiently transfected into COS7 cells. On the next day, fluorescence was detected by an electron multiplying CCD camera (iXON plus, ANDOR Technology Ltd., US) with a Plan-Apochromat $63 \times / 1.40$ Oil DIC M27 objective lens equipped to structured illumination microscopy (ELYRA, Carl Zeiss, Germany). To activate PPAP1.1 and to take an image, the whole region of the image was irradiated for $2.5 \mathrm{~s}$ with an optically pumped semiconductor laser $(488 \mathrm{~nm})$. An image of lifeact-mCherry was then taken sequentially with an optically pumped semiconductor laser $(561 \mathrm{~nm})$ (acquisition time $2.5 \mathrm{~s}$ ), followed by $15 \mathrm{~s}$ in the dark. This cycle (total $20 \mathrm{~s}$ ) was repeated. The overlay images in Figs. 4C and 4F were prepared by MetaMorph (Molecular Devices Japan, Tokyo, Japan).

\section{Statistics}

All values are expressed as mean \pm S.E.M. Statistical analysis was performed using the Student's $t$-test. A statistical analysis of $\mathrm{PIP}_{3}$-dependent changes in the ratio of the membrane area was performed by Prism (GraphPad Software, La Jolla, CA).

\section{Results and Discussion}

Cryptochrome 2 (CRY2) is a plant photoreceptor that regulates plant functions, such as flowering ${ }^{23}$ and the circadian rhythm. ${ }^{24}$ 
A
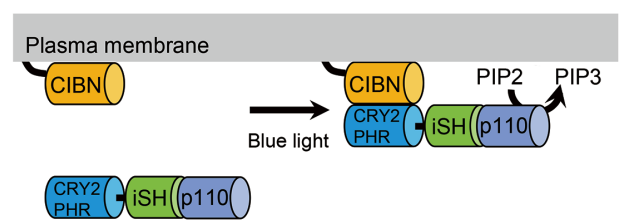

B

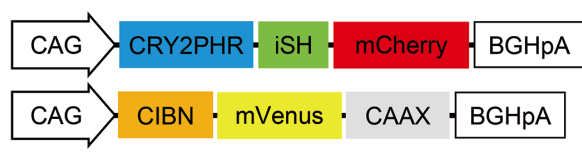

C
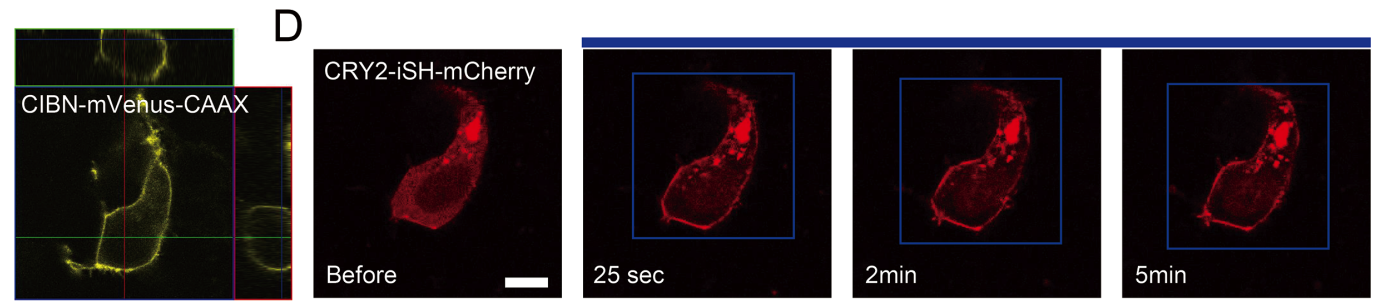

$E$

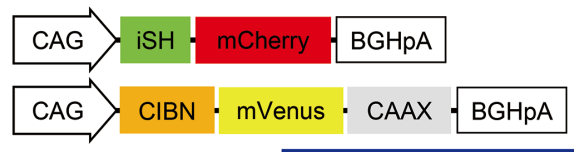

$\mathrm{F}$

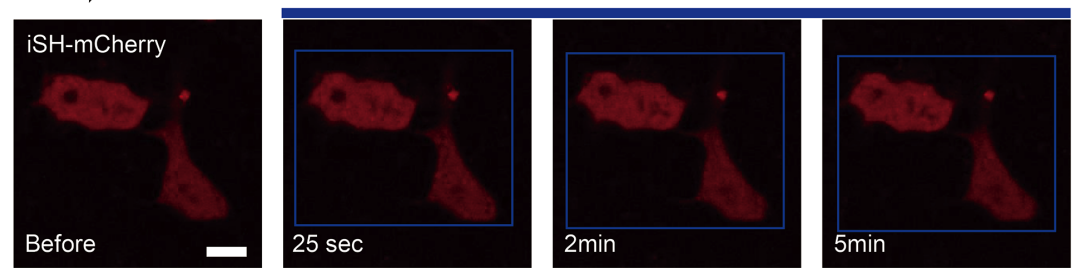

Fig. 1 Principle of the PAPP1.0 system. (A) PAPP1.0 consists of CRY2PHR and CIBN photodimerizer. CRY2PHR tagged with the inter-SH domain that binds to the endogenous PI3K catalytic domain, p110, is expressed in the cytosolic fraction. Additionally, CIBN was tethered to the plasma membrane through the CAAX motif of K-Ras. In response to blue light, CRY2PHR is recruited to the plasma membrane through CIBN-CRY2PHR association. According to this response, the p110 subunit also approaches the plasma membrane. This p110 subunit generates $\mathrm{PIP}_{3}$ from phosphatidylinositol 4,5-bisphosphate $\left(\mathrm{PIP}_{2}\right)$. (B) The cDNA construct of PAPP1.0. Both constructs were integrated downstream of the CAG promoter. (C) Confirmation of the appropriate localization of CIBN-mVenusCAAX visualized by mVenus fluorescence under laser scanning microscopy (LSM710, Carl Zeiss). (D) Time-course observation of changes in CRY2-iSH-mCherry localization. As blue light irradiation protocol, the region in a blue square in the whole image was irradiated for $11.40 \mathrm{~s}$ with blue light at $0.5 \mathrm{~W} / \mathrm{m}^{2}$. Next, the image was sequentially taken, followed by in a dark condition. This sequential procedure (total $19.2 \mathrm{~s}$ ) was one cycle, and repeated. White bar $=10 \mu \mathrm{m}$. (E) A control cDNA variant of PPAP1.0 in which CRY2PHR was removed. (F) Time-course analysis of the PPAP1.0 control variant. As blue light irradiation protocol, the region in a blue square in the whole image was irradiated for $14.3 \mathrm{~s}$ with blue light at $0.5 \mathrm{~W} / \mathrm{m}^{2}$. Next, the image was sequentially taken, followed by in dark condition. This sequential procedure (total $22.1 \mathrm{~s}$ ) was one cycle, and repeated. White bar $=10 \mu \mathrm{m}$.

CRY2 conveys signals through homodimerization and binding with CRY2-interacting basic helix-loop-helix1 (CIB1) in a blue light-dependent manner. We harnessed CRY2-CIB1 dimerization for $\mathrm{PIP}_{3}$ production (Fig. 1A). In order to improve the expression of CIB1 (335 aa), we employed the N-terminal truncated CIB1 (CIBN: 170 aa) for our construct in this study, which attaches at the plasma membrane through the CAAX domain of K-Ras. ${ }^{25}$ Additionally, CRY2PHR (498 aa), which is also a truncated version of full-length CRY2, was used. CRY2PHR was fused with the inter-SH domain (CRY2-iSH), which is a regulatory subunit of PI3K and binds the catalytic domain (p110 subunit) of endogenous PI3K. ${ }^{14}$ CRY2-iSH was expressed in the cytosolic fraction in cells. Upon blue light irradiation, PI3K approaches the plasma membrane due to the CRY2PHR-CIBN interaction, and $\mathrm{PIP}_{3}$ is generated from phosphatidylinositol 4,5-bisphosphate $\left(\mathrm{PIP}_{2}\right)$ (Fig. 1A). We named this CRY2PHR-CIBN system as PPAP1.0 ( $\mathrm{PIP}_{3}$ production by photo-activated PI3K).

To examine whether PPAP1.0 functions appropriately, we co-expressed CRY2PHR and CIBN-CAAX constructs (Fig. 1B) in HEK293 cells. One day after transfection, we confirmed that mVenus (a yellow fluorescent protein)-tagged CIBN-CAAX was properly localized at the plasma membrane (Fig. 1C), and that mCherry (a red fluorescent protein)-tagged CRY2PHR-iSH was expressed in the cytosolic fraction in HEK293 cells (Fig. 1D, "Before"). Some CRY2PHRs were aggregated due to CRY2 homodimerization. Upon blue light irradiation, CRY2PHR-iSH-mCherry was promptly translocated to the plasma membrane (Fig. 1D and Supplemental movie 1). In contrast, iSH-mCherry as a control construct (Fig. 1E) did not translocate to the plasma membrane even after blue light irradiation (Fig. 1F), indicating that CRY2PHR translocation 
A

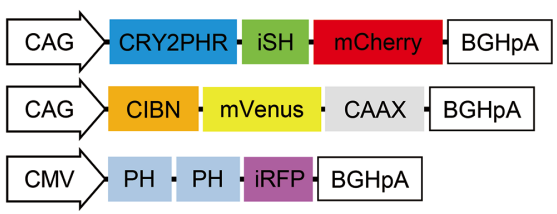

B

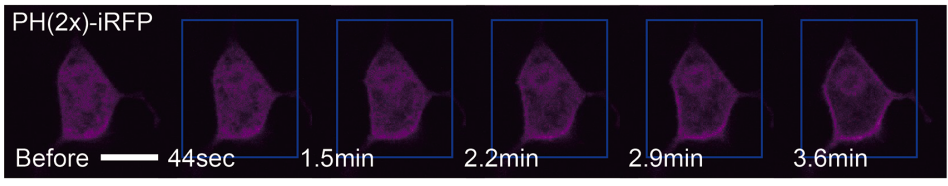

C

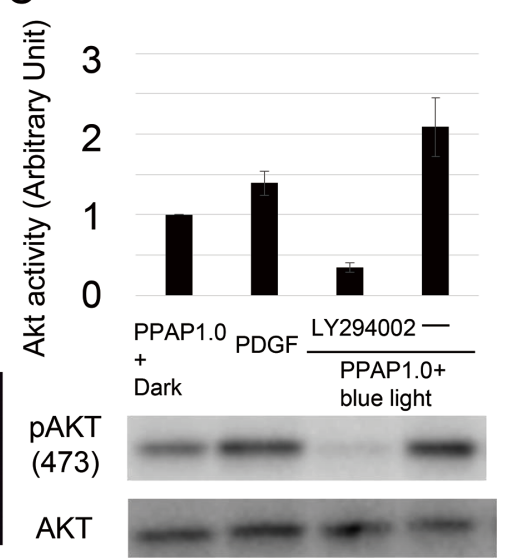

Fig. 2 PAPP1.0-induced $\mathrm{PIP}_{3}$ production. (A) cDNA construct of the probe to detect $\mathrm{PIP}_{3}(\mathrm{PH}(\times 2)$ iRFP) and PPAP1.0. (B) Time-course of $\mathrm{PIP}_{3}$ production detected by the $\mathrm{PIP}_{3}$ probe upon blue light irradiation in NIH3T3 cells expressing PPAP1.0. As blue light irradiation protocol, the region in a blue square in the whole image was irradiated for $12 \mathrm{~s}$ with blue light at $0.5 \mathrm{~W} / \mathrm{m}^{2}$. Next, the image was sequentially taken, followed by a dark condition. This sequential procedure (total $43.5 \mathrm{~s}$ ) was one cycle, and repeated. White bar $=20 \mu \mathrm{m}$. (C) Measurement of Akt activity based on Akt phosphorylation by western blotting after PPAP1.0 was irradiated with blue light $\left(10 \mathrm{~min}, 1 \mathrm{~W} / \mathrm{m}^{2}\right)$. Akt activity was calculated as follows. The intensity values of the pAkt and Akt bands on western blotting were measured using an ImageJ software (NIH). The pAkt (serine residue 473) intensity values were normalized by Akt ones of each lane. Then, each normalized pAkt intensity value was divided by the one of PPAP1.0+Dark as Akt activity.

was caused by the CRY2PHR-CIBN interaction. This data indicates that the CRY2PHR-CIBN system functions appropriately in a blue light-dependent manner.

Next, we checked whether $\mathrm{PIP}_{3}$ was clearly produced in response to the CRY2PHR-CIBN interaction after blue light irradiation. CRY2PHR and CIBN constructs were expressed in NIH3T3 cells together with a $\mathrm{PH}(\times 2)$-iRFP construct as a $\mathrm{PIP}_{3}$ biosensor, which consists of iRFP and tandem pleckstrin homology (PH) domains derived from Akt (Figs. 2A and 2B). After blue light irradiation, $\mathrm{PH}(\times 2)$-iRFP was translocated to the plasma membrane within 5 min, just as well as CRY2PHRiSH-mCherry, indicating $\mathrm{PIP}_{3}$ production at the plasma membrane (Fig. 2B). This $\mathrm{PIP}_{3}$ generation was also checked by endogenous Akt phosphorylation at the serine 473 position (Fig. 2C), because $\mathrm{PIP}_{3}$ production induces Akt phosphorylation. ${ }^{26}$ Ten minutes after blue light irradiation, PPAP1.0 generated 2.1 times more $\mathrm{PIP}_{3}$ than PPAP1.0 in the dark, and 6.1-times more $\mathrm{PIP}_{3}$ than under the condition in the presence of the PI3K inhibitor LY294002. Additionally, the PPAP1.0-induced Akt phosphorylation was more than that induced by PDGF stimulation, which activates Akt through PDGF receptor/PI3K on the plasma membrane (PDGF stimulation induced 4.1 times $\mathrm{PIP}_{3}$ production compared to the condition with LY294002). These data indicated that PPAP1.0 robustly induces $\mathrm{PIP}_{3}$ production in a blue light-dependent manner, and the extent of PPAP1.0-induced $\mathrm{PIP}_{3}$ increase falls into the physiological range in the previous studies that are described in introduction section. 9,27

Next, we examined whether PPAP1.0 can induce ruffles and lamellipodia in a blue light-dependent manner. PPAP1.0 was expressed in COS7 cells (Figs. 3A and 3B), which often exhibits ruffles and lamellipodia at the plasma membrane. A restricted cell membrane region was irradiated with blue light (blue square in Fig. 3B), causing CRY2-iSH-mCherry to accumulate at that region (white arrows in Fig. 3B, lower panels), and COS7 cells gradually moved toward the blue square region (Supplemental movie 2). At nearly the same time, ruffles disappeared at the lower region of COS7 cells and concentrated at the upper region (white arrow heads in Fig. 3B, upper panels). In contrast, the deletion construct of the iSH domain of PPAP1.0 (Fig. 3C) did not change COS7, although CRY2-mCherry accumulated at the blue light irradiated region (white arrows in Fig. 3D). We evaluated the extent of cell motility as cell membrane area increase ratio, in which cell membrane area in the blue square region after blue light irradiation was divided by the one before blue light irradiation. As a result, 8 out of 10 COS7 cells expressing PPAP1.0 showed higher ratio than 1.0 (Fig. 3E). The reason why cell motility was not induced in 2 COS7 cells is speculated to be as follows. PIP 3 is not evenly distributed at the cell membrane because almost all of adherent cells migrate persistently or randomly, ${ }^{28}$ and basically accumulates at the front of the migration. ${ }^{29}$ When the opposite side of the region where $\mathrm{PI} 3 \mathrm{~K} / \mathrm{PIP}_{3}$ is already accumulated was irradiated with blue light, the efficiency of $\mathrm{PIP}_{3}$ production was reduced due to the lack of PI3K on site, leading to less cell motility. Another possibility is that cell motility is regulated by not only $\mathrm{PIP}_{3}$, but also other factors, such as $\mathrm{Rac}^{30}$ and ERK. ${ }^{31}$ These factors could dominantly control cell motility more than $\mathrm{PIP}_{3}$ depending on the situation. Taken together, these data suggest that $\mathrm{PIP}_{3}$ controls cell motility and membrane ruffles.

Since membrane dynamics is regulated by actin polymerization/ depolymerization, we tried to image $\mathrm{PIP}_{3}$-dependent actin dynamics during the plasma membrane expansion. To observe detailed actin movement, we used structured illumination microscopy (SIM), which allowed us to observe it with high spatial resolution. ${ }^{32}$ Additionally, in order to visualize actin dynamics, we used mCherry-tagged lifeact that is comprised of 17-amino-acid peptide, and specifically binds to filamentous actin structures $^{33,34}$ (Fig. 4A). Furthermore, we minimized the number of constructs transfected into cells, which avoided the 
A

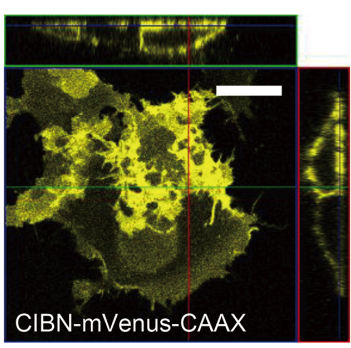

B
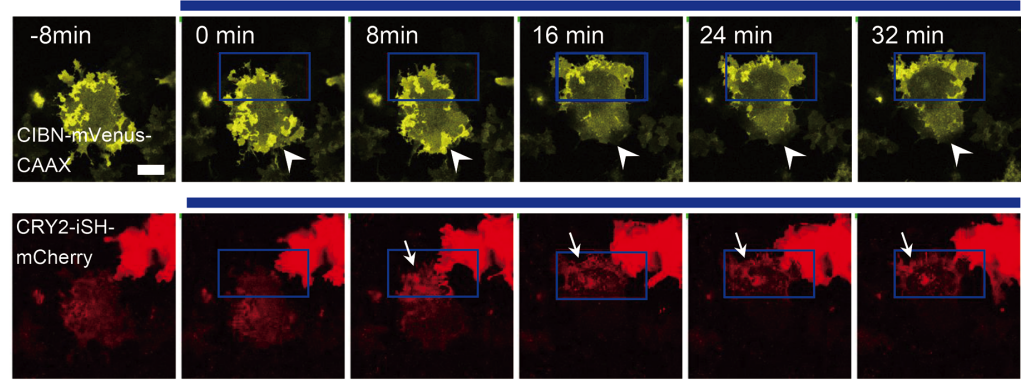
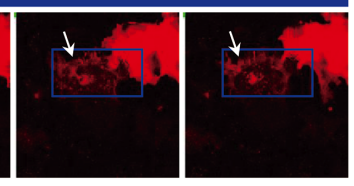

C

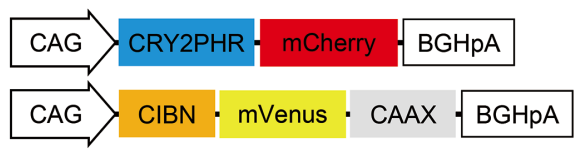

CIBN-mVenus-CAAX

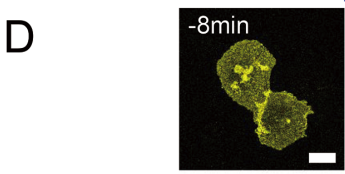

CRY2-mCherry
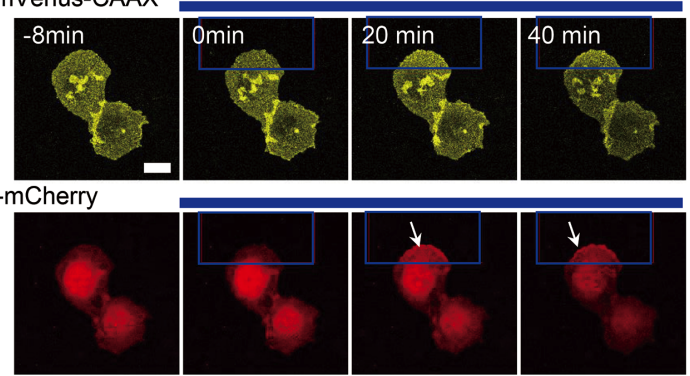

$\mathrm{E}$

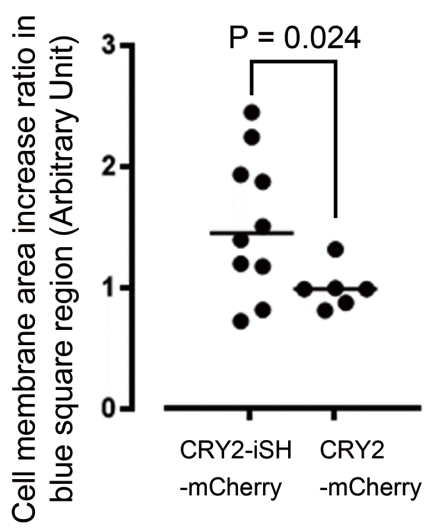

Fig. 3 Investigation of PPAP1.0-induced cell motility and plasma membrane ruffles. (A) CIBNmVenus-CAAX localization at the plasma membrane. White bar $=20 \mu \mathrm{m}$. (B) Time-course analysis of cell motility and ruffles in COS7 cells expressing PPAP1.0 after blue light irradiation. As blue light irradiation protocol, the region in a blue square in a COS7 cell was irradiated for $5.43 \mathrm{~s}$ with blue light at $0.5 \mathrm{~W} / \mathrm{m}^{2}$. Next, an image was sequentially taken, followed by the dark condition. This sequential procedure (total $44.4 \mathrm{~s}$ ) was one cycle, and repeated. White bar $=20 \mu \mathrm{m}$. (C) Control optogenetic tool for PPAP1.0 in which CRY2PHR was deleted was transfected in COS7 cells. (D) Time-course observation of cell motility and ruffles in COS7 cells expressing control PPAP1.0 lacking the iSH domain after blue light irradiation. As blue light irradiation protocol, the region in a blue square in the whole view of the image was irradiated for $10 \mathrm{~s}$ with blue light at $0.5 \mathrm{~W} / \mathrm{m}^{2}$. Next, the image was sequentially taken, followed by the dark condition. This sequential procedure (total $41.8 \mathrm{~s}$ ) was one cycle, and repeated. (E) Analysis of the plasma membrane area increase. The plasma membrane area after blue light irradiation in the blue square region of a COS7 cell was normalized by one before blue light irradiation as cell membrane area increase value. $P$-value was calculated by Student's $t$-test (see Experimental section, Statistics).

situation that the expression level of PPAP is reduced. CRY2PHR-iSH and CIBN-mVenus-CAAX were linked by the P2A/T2A sequence (Fig. 4A), by which both constructs were separated after translation, ${ }^{35}$ which we termed as PPAP1.1. These constructs were expressed in COS7 cells (Fig. 4B), with actin fibers shown as pseudo-color images. Upon blue light irradiation to the whole cell region, the plasma membrane expansion occurred at almost all cell edges (Fig. 4C). During the plasma membrane expansion, actin fibers dynamically changed. Interestingly, we observed (white dotted box in Fig. 4B, and white arrows in Fig. 4D) that a single actin fiber protruded during 7-9 min out of the plasma membrane by polymerization, receded to the plasma membrane during 10 $11 \mathrm{~min}$, and was finally taken up into the plasma membrane without depolymerization (white arrows in Fig. 4D, and Supplemental movie 3).

Our previous report found that T-lymphoma invasion and metastasis 1 (Tiam1), which is a GEF regulating Rac1 activation, induces apical ruffles, with ruffle size of $\sim 4 \mu \mathrm{m}$ and lifetime of $\sim 2$ min. ${ }^{36}$ We investigated whether $\mathrm{PIP}_{3}$-generated apical ruffles can be observed. In COS7 cells, which provide less plasma membrane expansion in response to blue light (Fig. 4F), we found $\mathrm{PIP}_{3}$-dependent apical ruffles (Figs. $4 \mathrm{E}$ and $4 \mathrm{~F}$ ). Unlike Tiam1-dependent apical ruffles, the lifetime of $\mathrm{PIP}_{3}$-dependent apical ruffles is longer ( $20 \mathrm{~min})$, yet the ruffle size is the same $(4-5 \mu \mathrm{m})$ (white arrows in Fig. 4G, Supplemental movie 4). These data indicate that there are various apical ruffles that are dependent on different signaling molecules.

\section{Conclusions}

We constructed PPAP1.0 and PPAP1.1, which quickly produce $\mathrm{PIP}_{3}$ at local regions in living cells in response to blue light. Using PPAP1.0, we revealed that $\mathrm{PIP}_{3}$ controls cell motility as well as membrane ruffles. Combined with SIM, PPAP1.1 allowed us to visualize detailed $\mathrm{PIP}_{3}$-induced membrane dynamics with high spatial and temporal resolution. This enabled us to observe unique actin fiber movements during lamellipodia and apical ruffle formation. 


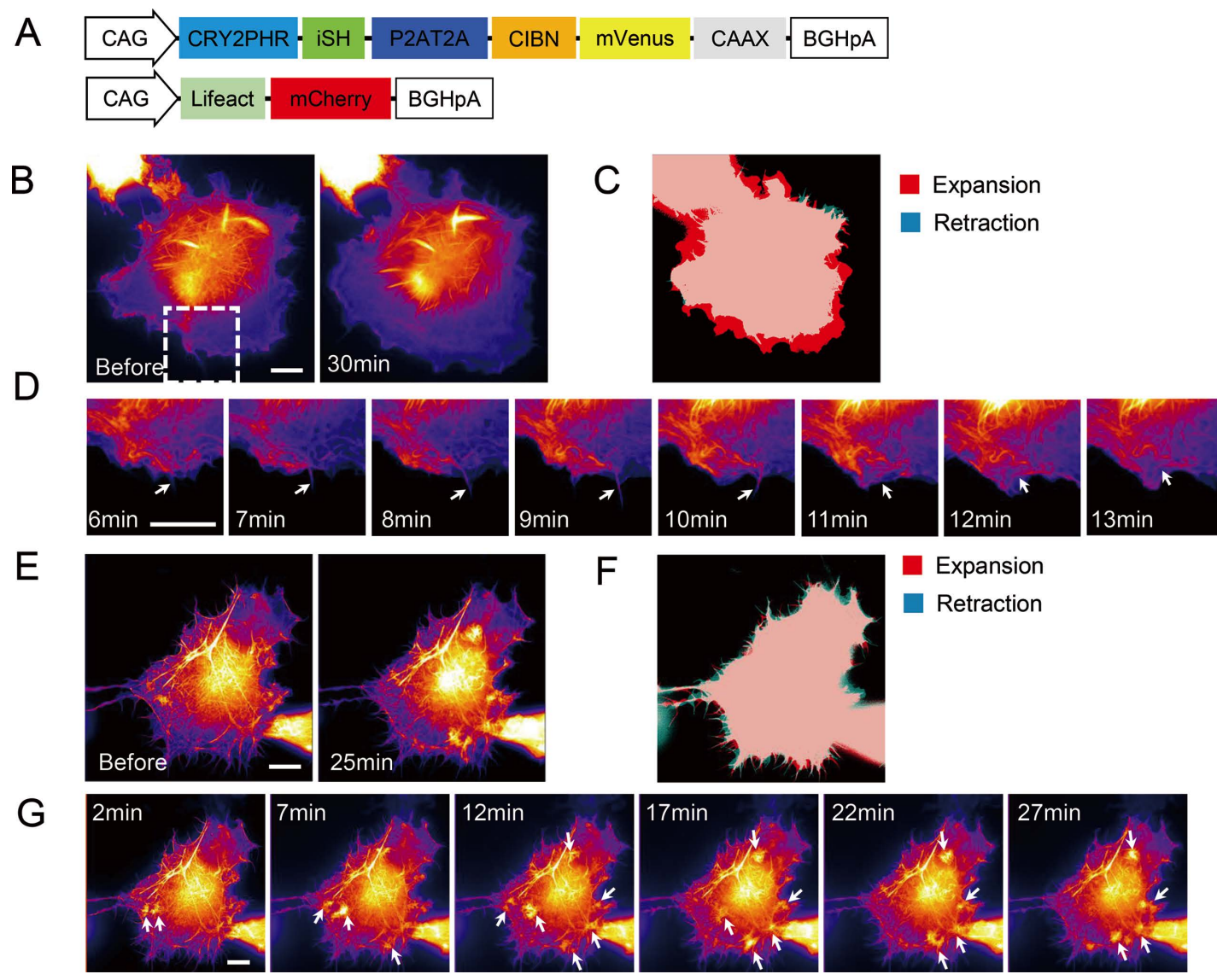

Fig. 4 Actin dynamics observation in COS7 cells expressing PPAP1.1 using SIM. (A) cDNA constructs of PPAP1.1. PPAP1.1 includes both CRY2PHR and CIBN units in the pCAG vector. The lifeact-mCherry construct was subcloned in the downstream of the CMV promoter in a pcDNA3.1(+) vector. (B) Pseudo-color images of actin fibers taken by SIM. To activate PPAP1.1 and take the image, whole region of the image was irradiated for $2.5 \mathrm{~s}$ with blue light $(488 \mathrm{~nm})$, and then an image of lifeact-mCherry was taken sequentially, followed by $15 \mathrm{~s}$ in the dark. This cycle (total $20 \mathrm{~s}$ ) was repeated. White bar $=10 \mu \mathrm{m}$. (C) Kymography of membrane expansion and retraction. Red and blue colors indicate expanded and retracted areas $30 \mathrm{~min}$ after blue light irradiation compared to before irradiation, respectively. (D) Time-course analysis of actin fibers during blue light irradiation. White arrows in the panels indicate a single actin fiber. White bar $=10 \mu \mathrm{m}$. (E) Apical ruffles in COS7 cells expressing PPAP1.1. White bar $=10 \mu \mathrm{m}$. (F) Kymograph of membrane expansion and retraction. (G) Time-course observation of apical ruffles (shown with white arrows) during blue light irradiation. White bar $=10 \mu \mathrm{m}$.

\section{Acknowledgements}

This work was supported by CREST, JST (to M. S.) and PRIME from Japan Agency for Medical Research and Development (to Y. U.).

\section{Supporting Information}

Supplemental movie 1: Time course of the translocation ofCRY2PHR-iSH-mCherry in HEK293 cells after blue light irradiation. Supplemental movie 2: Time course of directional moving of COS7 cells after local blue light irradiation. Supplemental movie 3: Time course of membrane expansion and lamellipodia of COS7 cells after blue light irradiation. Supplemental movie 4: Time course of apical ruffles of COS7 cells after blue light irradiation. This material is available free of charge on the Web at http://www.jsac.or.jp/analsci/.

\section{References}

1. E. S. Chhabra and H. N. Higgs, Nat. Cell Biol., 2007, 9, 1110.

2. S. J. Heasman and A. J. Ridley, Nat. Rev. Mol. Cell Biol., 2008, 9, 690.

3. R. H. Insall and O. D. Weiner, Dev. Cell, 2001, 1, 743.

4. T. Oikawa, H. Yamaguchi, T. Itoh, M. Kato, T. Ijuin, D. Yamazaki, S. Suetsugu, and T. Takenawa, Nat. Cell Biol., 2004, 6, 420.

5. M. Shinohara, Y. Terada, A. Iwamatsu, A. Shinohara, N. Mochizuki, M. Higuchi, Y. Gotoh, S. Ihara, S. Nagata, H. Itoh, Y. Fukui, and R. Jessberger, Nature, 2002, 416, 759.

6. Y. Ueda, Mol. Neurobiol., 2014, 50, 821.

7. Y. Ueda and Y. Hayashi, J. Neurosci., 2013, 33, 11040.

8. K. L. Arendt, M. Royo, M. Fernandez-Monreal, S. Knafo, C. N. Petrok, J. R. Martens, and J. A. Esteban, Nat. Neurosci., 2010, 13, 36.

9. H. Ogiso and R. Taguchi, Anal. Chem., 2008, 80, 9226. 
10. H. H. Bui, P. E. Sanders, D. Bodenmiller, M. S. Kuo, G. P. Donoho, and A. S. Fischl, Anal. Biochem., 2018, 547, 66.

11. A. C. Newton, J. Lipid Res., 2009, 50(Suppl), S266.

12. J. Han, K. Luby-Phelps, B. Das, X. Shu, Y. Xia, R. D. Mosteller, U. M. Krishna, J. R. Falck, M. A. White, and D. Broek, Science, 1998, 279, 558.

13. M. Innocenti, E. Frittoli, I. Ponzanelli, J. R. Falck, S. M. Brachmann, P. P. Di Fiore, and G. Scita, J. Cell Biol., 2003, 160, 17.

14. T. Kakumoto and T. Nakata, PLoS One, 2013, 8, e70861.

15. M. Sato, Y. Ueda, T. Takagi, and Y. Umezawa, Nat. Cell Biol., 2003, 5, 1016.

16. J. Jaworski, S. Spangler, D. P. Seeburg, C. C. Hoogenraad, and M. Sheng, J. Neurosci., 2005, 25, 11300.

17. Y. Ueda and M. Sato, ChemBioChem, 2018, 19, 1217.

18. Y. Nihongaki, H. Suzuki, F. Kawano, and M. Sato, ACS Chem. Biol., 2014, 9, 617.

19. G. Yu, H. Onodera, Y. Aono, F. Kawano, Y. Ueda, A. Furuya, H. Suzuki, and M. Sato, Sci. Rep., 2016, 6, 35777.

20. Y. Nihongaki, S. Yamamoto, F. Kawano, H. Suzuki, and M. Sato, Chem. Biol., 2015, 22, 169.

21. Y. Nihongaki, Y. Furuhata, T. Otabe, S. Hasegawa, K. Yoshimoto, and M. Sato, Nat. Methods, 2017, 14, 963.

22. J. Miyazaki, S. Takaki, K. Araki, F. Tashiro, A. Tominaga, K. Takatsu, and K. Yamamura, Gene, 1989, 79, 269.

23. M. Endo, N. Mochizuki, T. Suzuki, and A. Nagatani, Plant. Cell., 2007, 19, 84.

24. P. A. Salome, J. P. To, J. J. Kieber, and C. R. McClung,
Plant. Cell., 2006, 18, 55.

25. A. Apolloni, I. A. Prior, M. Lindsay, R. G. Parton, and J. F. Hancock, Mol. Cell Biol., 2000, 20, 2475.

26. J. Downward, Curr. Opin. Cell Biol., 1998, 10, 262.

27. J. Clark, K. E. Anderson, V. Juvin, T. S. Smith, F. Karpe, M. J. Wakelam, L. R. Stephens, and P. T. Hawkins, Nat. Methods, 2011, 8, 267.

28. M. Yamao, H. Naoki, K. Kunida, K. Aoki, M. Matsuda, and S. Ishii, Sci. Rep., 2015, 5, 17527.

29. T. Nishioka, K. Aoki, K. Hikake, H. Yoshizaki, E. Kiyokawa, and M. Matsuda, Mol. Biol. Cell, 2008, 19, 4213.

30. Y. I. Wu, D. Frey, O. I. Lungu, A. Jaehrig, I. Schlichting, B. Kuhlman, and K. M. Hahn, Nature, 2009, 461, 104.

31. K. Aoki, Y. Kondo, H. Naoki, T. Hiratsuka, R. E. Itoh, and M. Matsuda, Dev. Cell., 2017, 43, 305.

32. Y. Hirano, A. Matsuda, and Y. Hiraoka, Microscopy (Oxf), 2015, 64, 237.

33. J. Riedl, A. H. Crevenna, K. Kessenbrock, J. H. Yu, D. Neukirchen, M. Bista, F. Bradke, D. Jenne, T. A. Holak, Z. Werb, M. Sixt, and R. Wedlich-Soldner, Nat. Methods, 2008, 5, 605 .

34. S. Nishi, C. Yamamoto, S. Yoneda, and S. Sueda, Anal. Sci., 2017, 33, 897.

35. J. H. Kim, S. R. Lee, L. H. Li, H. J. Park, J. H. Park, K. Y. Lee, M. K. Kim, B. A. Shin, and S. Y. Choi, PLoS One, 2011, 6, e18556.

36. A. Furuya, F. Kawano, T. Nakajima, Y. Ueda, and M. Sato, ACS Synth. Biol., 2017, 6, 1086. 